\title{
Geometric Phase of Three-level Systems in Interferometry
}

\author{
Barry C. Sanders ${ }^{1}$ Hubert de Guise, ${ }^{1,2}$ Stephen D. Bartlett, ${ }^{1}$ and Weiping Zhang ${ }^{1}$ \\ ${ }^{1}$ Department of Physics, Macquarie University, Sydney, New South Wales 2109, Australia \\ ${ }^{2}$ Centre de Recherche Mathématique, Université de Montréal, \\ C.P. 6128-A, Montréal, Québec H3C 3J7, Canada
}

(Dated: November 14, 2000)

\begin{abstract}
We present the first scheme for producing and measuring an Abelian geometric phase shift in a three-level system where states are invariant under a non-Abelian group. In contrast to existing experiments and proposals for experiments, based on U(1)-invariant states, our scheme geodesically evolves $\mathrm{U}(2)$-invariant states in a four-dimensional $\mathrm{SU}(3) / \mathrm{U}(2)$ space and is physically realized via a three-channel optical interferometer.
\end{abstract}

PACS numbers: 03.65.Bz, 02.20.-a, 42.50.-p

Cyclic evolution of a wave function yields the original state plus a phase shift, and this phase shift is a sum of a dynamical phase and a geometric (or topological, or quantal, or Berry) phase shift [1, 2]. The geometric phase shift is important, not just for quantum systems, but also for all of wave physics. Thus far, controlled geometric-phase experiments, both realized and proposed, have been exclusively concerned with the so-called Abelian geometric phase arising in the evolution of U(1)-invariant states, for example, states of the Poincaré sphere (in the case of $\mathrm{SU}(2)$ states) [3, 田, the Lobachevsky plane [5] (in the case of $\mathrm{SU}(1,1)$ states) and $\mathbb{R}^{2}$ (for the Aharonov-Bohm phase) [1]. Here we introduce an optical scheme to produce and detect an Abelian geometric phase shift which arises from geodesic transformations of $\mathrm{U}(2)$-invariant states in a four-dimensional $\mathrm{SU}(3) / \mathrm{U}(2)$ space. This scheme employs a three-channel optical interferometer and four experimentally adjustable parameters to observe the geometric phase in its full generality.

Geometric phases in SU(3) systems have been the subjects of recent mathematical studies [6, 7] and establish the geometric phase shift expected for the cyclic evolution (up to a phase) of a three-level system. We propose to obtain this evolution using an interferometer as a sequence of unitary transformations given by optical elements. An optical SU(3) transformation can be realized by a three-channel optical interferometer 8 . The space of output states of the interferometer can be identified with $\mathrm{SU}(3) / \mathrm{U}(2)$, and will be referred to as the geometric space; this space is a generalization of the Poincaré sphere to a three-level system [7]. By adjusting the parameters of the interferometer, the output state can be made to evolve cyclically, up to a phase, through a triangle in the geometric space. The output of the interferometer may be any state along a path in $\mathrm{SU}(3) / \mathrm{U}(2)$, determined by fixing the four free parameters of the interferometer.

It is important to distinguish the evolution of states in the geometric space $\mathrm{SU}(3) / \mathrm{U}(2)$ from the transformations of the optical beam as it progresses through the interferometer. It will be shown later how the dynam- ical phase associated with these optical transformations can be eliminated. The cyclic evolution described in this paper occurs in the geometric space, and the geometric phase of interest is related to this evolution. We provide here the essential elements to obtain this evolution as well as to explain how to design the interferometer.

It is sufficiently general to consider the input state $\psi^{\text {in }}$ of a photon into one of the three input ports and the vacuum state into the other two ports. The parameters of the interferometer can be initially set such that the resultant $\mathrm{SU}(3)$ transformation is the identity, and thus the interferometer output state $\psi^{(1)}$ is also one photon at the corresponding output port and the vacuum at the two other ports. These parameters can then be adjusted to evolve the output state along a trajectory in the geometric space; this evolution may involve both a 'dynamical' phase shift and a geometric phase shift. Care must be taken when interpreting the adjective 'dynamical'. The output state does not evolve according to Schrödinger dynamics but instead follows a path in the geometric space parametrized by an evolution parameter $s$, which is a function of the adjustable parameters of the interferometer.

The dynamical and geometric phase shift contributions must be separated to obtain the geometric phase. A special role is played by geodesic evolution [9]; by transforming the output state along geodesic paths in the geometric space, the geometric phase shift along each path is zero. Thus, we consider three arbitrary states $\left\{\psi^{(k)} ; k=1,2,3\right\}$ in the geometric space which define a geodesic triangle (i.e., with sides given by the unique geodesics connecting these states). The parameters of the interferometer are adjusted to evolve the output state along this general geodesic triangle $\psi^{(1)} \rightarrow \psi^{(2)} \rightarrow$ $\psi^{(3)} \rightarrow \psi^{(4)}=e^{i \varphi_{g}} \psi^{(1)}$, where $\varphi_{g}$ is the total geometric phase gained by cyclic evolution and depends on four free parameters of the interferometer. Fig. 1 1 gives a diagrammatic depiction of this scheme.

The evolution of the state $\psi^{(1)}$ to the state $\psi^{(4)}=$ $e^{i \varphi_{g}} \psi^{(1)}$ via three geodesic paths in the geometric space can be described by three one-parameter SU(3) group 


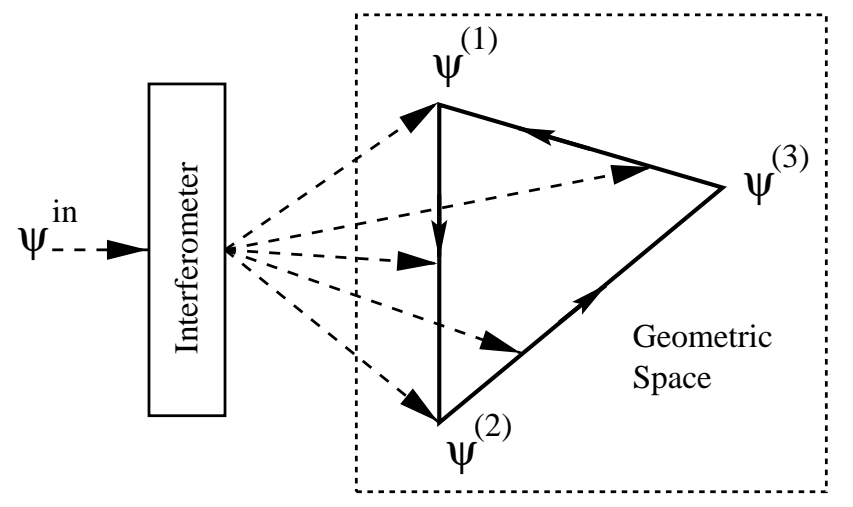

FIG. 1: The geodesic evolution in the geometric space is depicted diagrammatically. By adjusting the parameters of the interferometer, the output state in the geometric space can be made to evolve along geodesic paths, from one vertex to the next, until the triangle is closed. The SU(3) transformations $U_{k}^{g}\left(s_{k}\right)$ map the output state along the geodesic paths in the geometric space.

elements $\left\{U_{k}^{g}\left(s_{k}\right) ; k=1,2,3\right\}$, with $s_{k}$ an evolution parameter. These transformations satisfy the conditions that $U_{k}^{g}(0)$ is the identity element and

$$
U_{k}^{g}\left(s_{k}^{0}\right) \psi^{(k)}=\psi^{(k+1)}, \quad k=1,2,3,
$$

for some fixed values $\left\{s_{k}^{0}\right\}$. It is always possible to choose unit vectors $\psi^{(k)}$ such that $\left\langle\psi^{(k+1)} \mid \psi^{(k)}\right\rangle$ is real and positive. We consider evolutions $U_{k}^{g}\left(s_{k}\right)$ of the form

$$
U_{k}^{g}\left(s_{k}\right)=V_{k} \cdot R_{s_{k}} \cdot V_{k}^{-1},
$$

with $V_{k}$ an element of SU(3) satisfying $\left\langle\psi^{(k)}\left|U_{k}^{g}\left(s_{k}\right)\right| \psi^{(k)}\right\rangle$ real and positive, and

$$
R_{s_{k}} \equiv\left(\begin{array}{ccc}
\cos s_{k} & -\sin s_{k} & 0 \\
\sin s_{k} & \cos s_{k} & 0 \\
0 & 0 & 1
\end{array}\right)
$$

The form of the one-parameter subgroup $R_{s_{k}}$ with real entries was guided by the definition of a geodesic curve between two states $\psi^{(k)}$ and $\psi^{(k+1)}$, which can be written in the form [7]

$$
\begin{aligned}
\psi\left(s_{k}\right)= & \psi^{(k)} \cos s_{k} \\
& +\frac{\left(\psi^{(k+1)}-\psi^{(k)}\left\langle\psi^{(k+1)} \mid \psi^{(k)}\right\rangle\right)}{\sqrt{1-\left\langle\psi^{(k+1)} \mid \psi^{(k)}\right\rangle^{2}}} \sin s_{k}
\end{aligned}
$$

with $0 \leq s_{k} \leq s_{k}^{0}=\arccos \left\langle\psi^{(k+1)} \mid \psi^{(k)}\right\rangle$. It is straightforward to show that any $U_{k}^{g}\left(s_{k}\right)$ of the form given by Eq. (2) satisfying $\left\langle\psi^{(k+1)} \mid \psi^{(k)}\right\rangle$ real and positive gives evolution along a geodesic curve in $\mathrm{SU}(3) / \mathrm{U}(2)$.
Consider the three states

$$
\begin{aligned}
\psi^{(1)} & =\left(\begin{array}{l}
1 \\
0 \\
0
\end{array}\right)=e^{-i \varphi_{g}} \psi^{(4)}, \quad \psi^{(2)}=\left(\begin{array}{c}
\cos s_{1}^{0} \\
\sin s_{1}^{0} \\
0
\end{array}\right), \\
\psi^{(3)} & =\left(\begin{array}{c}
\cos s_{1}^{0} \cos s_{2}^{0}-e^{i \alpha} \sin s_{1}^{0} \sin s_{2}^{0} \cos \beta \\
\sin s_{1}^{0} \cos s_{2}^{0}+e^{i \alpha} \cos s_{1}^{0} \sin s_{2}^{0} \cos \beta \\
\sin \beta \sin s_{2}^{0}
\end{array}\right),
\end{aligned}
$$

with $s_{1}^{0}, s_{2}^{0}, \alpha$ and $\beta$ arbitrary. These three states form the vertices of the geodesic triangle in the geometric space. They are sufficiently general to include all types of geodesic triangles [7].

Although the three-channel interferometer can be expressed as an $\mathrm{SU}(3)$ transformation (or sequence of $\mathrm{SU}(3)$ transformations), the optical elements of the interferometer are composed of beam splitters, mirrors and phase shifters. Provided that losses can be ignored, each of these optical elements can be associated with an $\mathrm{SU}(2)$ unitary transformation 10, 11]. It is therefore advantageous to factorize each $\mathrm{SU}(3)$ transformation into a product of $\mathrm{SU}(2)_{i j}$ subgroup transformations mixing fields $i$ and $j$ : first, an $\mathrm{SU}(2)_{23}$, followed by an $\mathrm{SU}(2)_{12}$ and completed by a final $\mathrm{SU}(2)_{23}$ transformation 112. Such a factorization makes the experimental design of the interferometer clear: fields 2 and 3 are mixed followed by a mixing of the output field 2 with the field in channel 1 , and, finally, the output field 2 is mixed with field 3 .

The $\mathrm{SU}(2)_{12}$ matrix $R_{s}$ in Eq. (3) is a special case of the generalized lossless beam splitter transformation for mixing channels 1 and 2. More generally a beam splitter can be described by a unitary transformation between two channels [11]. For example, a general $\mathrm{SU}(2)_{23}$ beam splitter transformation for mixing channels 2 and 3 is of the form

$$
R_{23}\left(\phi_{\mathrm{t}}, \theta, \phi_{\mathrm{r}}\right)=\left(\begin{array}{ccc}
1 & 0 & 0 \\
0 & e^{i \phi_{\mathrm{t}}} \cos \theta & -e^{-i \phi_{\mathrm{r}}} \sin \theta \\
0 & e^{i \phi_{\mathrm{r}}} \sin \theta & e^{-i \phi_{\mathrm{t}}} \cos \theta
\end{array}\right),
$$

with $\phi_{\mathrm{t}}$ and $\phi_{\mathrm{r}}$ the transmitted and reflected phase-shift parameters, respectively, and $\cos ^{2} \theta$ the beam splitter transmission. A generalized beam splitter can be realized as a combination of phase shifters and 50/50 beam splitters in a Mach-Zehnder interferometer configuration.

It is useful at this point to consider the nature of the geodesic transformations $U_{k}^{g}\left(s_{k}\right)$ and their realization in terms of optical elements. The interferometer can be adjusted to transform the input state $\psi^{\text {in }}$ to an arbitrary output state $\psi(s)$ anywhere along the geodesic triangle. This optical transformation can be related to an $\mathrm{SU}(3)$ transformation in the geometric space, which maps $\psi^{(1)}$ to $\psi(s)$ along a geodesic path. It is important to distinguish between the optical evolution through the interferometer from $\psi^{\text {in }}$ to $\psi(s)$, and the geodesic evolution in the geometric space from $\psi^{(1)}$ to $\psi(s)$. The goal of the following is to construct optical transformations in 


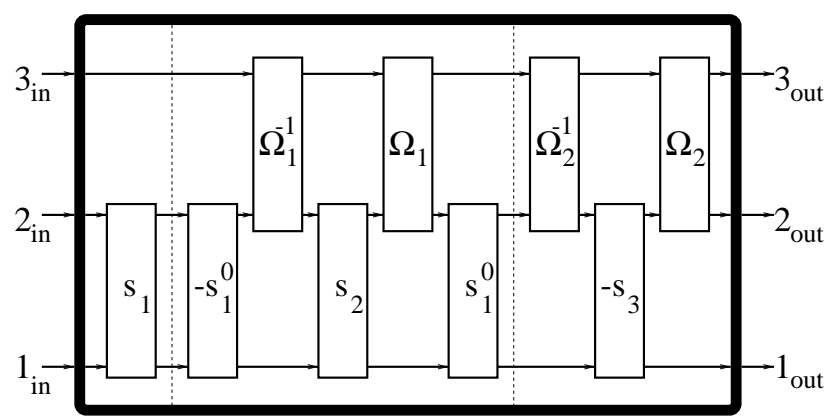

FIG. 2: The SU(3) interferometer is depicted, with three input ports $1_{\text {in }}, 2_{\text {in }}$ and $3_{\text {in }}$, and three output ports $1_{\text {out }}, 2_{\text {out }}$ and $3_{\text {out }}$. There are nine beam splitter transformations with parameters $s_{1}, s_{2}, s_{3}$ and $\Omega_{1}=(\alpha, \beta, 0), \Omega_{2}=(\chi, \tau,-\xi)$. For geodesic, cyclic evolution of the output state, only four parameters are independent.

terms of $\mathrm{SU}(2)$ elements which realize the geodesic evolution in the geometric space by appropriately adjusting parameters.

It will be convenient to express $\psi^{(3)}$ as $\left(e^{i \xi} \cos \eta, e^{i(\xi+\chi)} \sin \eta \cos \tau, \sin \eta \sin \tau\right)^{T}$, where $\xi, \quad \eta$, $\tau$ and $\chi$ are functions of $s_{1}^{0}, s_{2}^{0}, \alpha$ and $\beta$, the parameters of $\psi^{(3)}$ in Eq. (5). Following our factorization scheme, the geodesic evolution operators $U_{k}^{g}\left(s_{k}\right)$, connecting $\psi^{(k)}$ to $\psi^{(k+1)}$, can be expressed as

$$
\begin{aligned}
& U_{1}^{g}\left(s_{1}\right)=R_{s_{1}} \\
& U_{2}^{g}\left(s_{2}\right)=R_{s_{1}^{0}} \cdot R_{23}(\alpha, \beta, 0) \cdot R_{s_{2}} \cdot R_{23}^{-1}(\alpha, \beta, 0) \cdot R_{-s_{1}^{0}}, \\
& U_{3}^{g}\left(s_{3}\right)=R_{23}(\chi, \tau,-\xi) \cdot R_{-s_{3}} \cdot R_{23}^{-1}(\chi, \tau,-\xi)
\end{aligned}
$$

with $R_{s}$ given by Eq. (3), the parameters $s_{k}$ ranging from $0 \leq s_{k} \leq s_{k}^{0}$, and $s_{3}^{0}=\eta$. Note that $s_{3}^{0}$ and, in fact, all the parameters of $U_{3}^{g}\left(s_{3}\right)$ are fixed by the requirement that $\psi^{(4)}=e^{i \varphi_{g}} \psi^{(1)}$. Also note that, for each $k, U_{k}^{g}(0)$ is the identity in $\mathrm{SU}(3)$ and $U_{k}^{g}\left(s_{k}^{0}\right) \psi^{(k)}=\psi^{(k+1)}$ as required. Once it is observed that $\left\langle\psi^{(k+1)} \mid \psi^{(k)}\right\rangle=\cos s_{k}^{0}$, it is trivial to verify that each evolution satisfies Eq. (何) and is therefore geodesic.

The geometric phase for the cyclic evolution $\psi^{(1)} \rightarrow$ $\psi^{(4)}$ is given explicitly by

$$
\varphi_{g}=\xi=\arg \left(\cos s_{1}^{0} \cos s_{2}^{0}-e^{i \alpha} \sin s_{1}^{0} \sin s_{2}^{0} \cos \beta\right) .
$$

This phase depends on four free parameters in the experimental scheme: $s_{1}^{0}, s_{2}^{0}, \alpha$ and $\beta$, which describe a general geodesic triangle in $\mathrm{SU}(3) / \mathrm{U}(2)$.

The interferometer configuration for realizing the necessary evolution about the geodesic triangle is depicted in Fig. 2. This configuration consists of a sequence of $\mathrm{SU}(2)_{i j}$ transformations, which are physically realized by generalized beam splitters (e.g., Mach-Zehnder interferometers). We use the shorthand notation $\Omega_{i} \equiv\left(\alpha_{i}, \beta_{i}, \gamma_{i}\right)$ to designate the three parameters associated with a generalized beam splitter. The three-channel interferometer

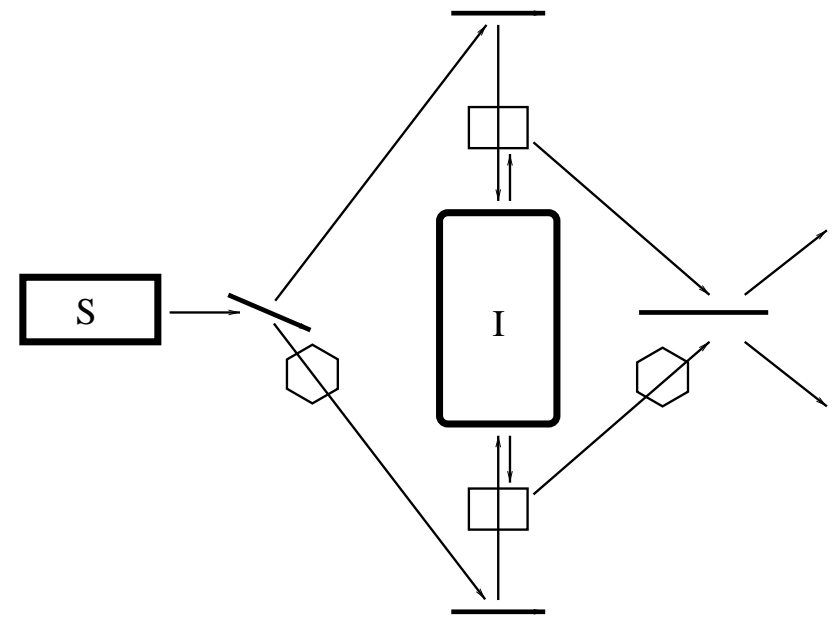

FIG. 3: In the interferometric scheme for detecting the geometric phase shift, the source (S) field is split by a $50 / 50$ beam splitter into two identically polarized output fields with equal amplitudes. The polarization of one output field is rotated to an orthogonal polarization, with the polarizer rotator represented by a hexagon. One field is injected into port $1_{\text {in }}$ of the SU(3) interferometer in Fig. 2, and the orthogonallypolarized field is injected into port $1_{\text {out }}$ at the other end of the interferometer. The output fields exit ports $1_{\text {out }}$ and $1_{\text {in }}$, respectively and are separated from the input fields by polarizing beam splitters at each of the two output ports, followed by mixing at a 50/50 beam splitter.

consists of a sequence of nine $\mathrm{SU}(2)_{i j}$ transformations. The field enters port $1_{\mathrm{in}}$, and the vacuum state enters ports $2_{\text {in }}$ and $3_{\text {in }}$. By adjusting the parameters of the interferometer, the output state can be made to evolve along the geodesic triangle $\psi^{(1)} \rightarrow \psi^{(4)}$ in the geometric space. We now consider how to measure the geometric phase as a function of the four free parameters $s_{1}^{0}, s_{2}^{0}, \alpha$ and $\beta$ describing a general geodesic triangle.

One check on the proper functioning of the interferometer is to place photodetectors at the ports $1_{\text {out }}, 2_{\text {out }}$, and $3_{\text {out }}$. The measured photodistribution for any output state can be compared to the predicted output $\psi(s)$ of the interferometer. In particular, for cyclic evolution to the output state $\psi^{(4)}$, there should be no photons exiting ports $2_{\text {out }}$ and $3_{\text {out }}$ regardless of the settings of the free parameters.

Consider the cyclic evolution of the output state to the state $\psi^{(4)}=e^{i \varphi_{g}} \psi^{(1)}$. A key technical challenge is measuring $\varphi_{g}$, because one must have a reference state with which to interfere the output state $\psi^{(4)}$. The input state $\psi^{\text {in }}$ is a poor choice, because the relationship between $\psi^{\text {in }}$ and $\psi^{(1)}$ involves an optical dynamical phase due to evolution through the interferometer. However, this optical phase can be eliminated through the use of a counter-progagating beam, described below.

A scheme for conducting such an experiment is depicted in Fig. 3. The source (a laser, for example) produces a polarized, stable, coherent beam of light 
which is split at a polarization-independent beam splitter. One beam travels to input port $1_{\text {in }}$ as shown in Fig. 2 and passes through the interferometer, exiting at output port $1_{\text {out }}$. The other beam is first "rotated" to an orthogonal polarization; it then enters port $1_{\text {out }}$ and counter-propagates through the interferometer, exiting at port $1_{\text {in }}$. The orthogonal polarizations of the two counter-propagating beams ensure that they do not interfere with each other inside the interferometer.

At the ports $1_{\text {in }}$ and $1_{\text {out }}$, there are polarizing beam splitters which deflect the outcoming beams but do not affect the propagation of the incoming beams. The output beams are directed to a beam splitter where they are made to interfere. The optical dynamical phase shift accumulated by each of the two counter-propagating beams through the SU(3) interferometer is identical, because of the time-reversal invariance of the Hamiltonian describing the evolution within the interferometer. Thus, the optical phase shifts cancel in the interference. Unitarity of the interferometer transformation guarantees that the geometric phase shift is $\varphi_{g}$ for one beam and $-\varphi_{g}$ for the other beam. Thus, the two beams interfere with relative phase $2 \varphi_{g}$.

By measuring the geometric phase $\varphi_{g}$ for various settings of the free parameters of the interferometer, it is possible to explore the geometric space with the most general geodesic triangles. The observed values can then be compared to the theoretical predictions.

If the source in Fig. 3 is a laser, operation at a low-light level can be undertaken to verify that the geometric phase shift is $\varphi_{g}$ even if the probability of more than one photon being present within the system is negligible. Low-light level operation, in the regime where the presence of more than one photon in the entire apparatus at any time is negligible, enables the testing of the geometric phase shift even when the discreteness of the field energy cannot be ignored [13].

A variation of the scheme in Fig. 3 can also be considered to verify that the geometric phase occurs for each photon. Kwiat and Chiao [4] conducted a measurement of geometric phase by employing parameteric down conversion (PDC), with a UV-pumped KDP crystal, to produce photon pairs. One photon undergoes a geometric phase shift, and the second photon in the pair is employed as a gate to register the event. By repeating this process for many 'single' photons, conditioned on detection of the gate photon, where the photon passes the first beam splitter in Fig. 3 and has an equal probabil- ity of propagating or, in an orthogonally polarized state, counter-propagating, through the three-channel interferometer, an interference pattern can be built up one photon at a time to establish that geometric phase is imposed one photon at a time, following Dirac's dictum that "each photon interferes only with itself" 14.

Although SU(3) interferometry has been considered in detail, the methods employed here can be extended to $\mathrm{SU}(\mathrm{N})$, or $\mathrm{N}$-channel, interferometry [8]. The schemes discussed above employing such a device would produce and enable observation of the geometric phase shift for geodesic transformations of states invariant under $\mathrm{U}(\mathrm{N}-1)$ subgroups of $\mathrm{SU}(\mathrm{N})$ states in the $2(\mathrm{~N}-1)$-dimensional coset space $\mathrm{SU}(\mathrm{N}) / \mathrm{U}(\mathrm{N}-1)$.

This work has been supported by two Macquarie University Research Grants and by an Australian Research Council Large Grant. BCS appreciates valuable discussions with J. M. Dawes and A. Zeilinger, and HdG acknowledges the support of Fonds F.C.A.R. of the Québec Government.

[1] M. V. Berry, Proc. Roy. Soc. (Lond.) 392, 45 (1984).

[2] B. Simon, Phys. Rev. Lett. 51, 2167 (1983); F. Wilczek and A. Shapere, Geometric Phases in Physics, Advanced Series in Mathematical Physics - Vol. 5 (World Scientific, Singapore, 1989).

[3] A. Tomita and R. Y. Chiao, Phys. Rev. Lett. 57, 937 (1986); R. Y. Chiao et al, Phys. Rev. Lett. 60, 1214 (1988); D. Suter et al, Phys. Rev. Lett. 60, 1218 (1988).

[4] P. G. Kwiat and R. Y. Chiao, Phys. Rev. Lett. 66, 588 (1991).

[5] R. Simon and N. Mukunda, Phys. Rev. Lett. 70, 880 (1993).

[6] N. Mukunda and R. Simon, Ann. Phys. (N.Y.) 228, 205 (1993); G. Khanna et al, Ann. Phys. (N.Y.) 253, 55 (1997).

[7] Arvind et al, J. Phys. A: Math. Gen. 30, 2417 (1997).

[8] M. Reck et al, Phys. Rev. Lett. 73, 58 (1994); B. C. Sanders et al, J. Phys. A: Math. Gen. 32, 7791 (1999).

[9] T. H. Chyba et al, Opt. Lett. 13, 562 (1988); R. Simon et al, Phys. Rev. Lett. 61, 19 (1988).

[10] B. Yurke et al, Phys. Rev. A 33, 4033 (1986).

[11] R. A. Campos et al, Phys. Rev. A 40, 1371 (1989).

[12] D. J. Rowe et al, J. Math. Phys. 40, 3604 (1999).

[13] P. Hariharan et al, J. Mod. Opt. 40, 871 (1993).

[14] P. A. M. Dirac, The Principles of Quantum Mechanics, 4th ed. (Oxford University Press, Oxford, 1958). 